\title{
Erratum: Ultrafast strong-field photoelectron emission due to two-color laser fields [Phys. Rev. B 98, 165442 (2018)]
}

Yi Luo and Peng Zhang

(Q) (Received 3 April 2019; published 18 April 2019)

DOI: 10.1103/PhysRevB.99.169907

We found errors in the legends of Figs. 2(c)-2(e) in the published version of our paper. The legend for the orange curve in Figs. 2(c)-2(e) should be $[0,1]$ instead of $[1,0]$. These corrections do not affect the other parts and the content of the paper. 